\title{
Higgs boson production at large transverse momentum within the SMEFT: analytical results
}

\author{
Massimiliano Grazzini ${ }^{1, a}$, Agnieszka Ilnicka ${ }^{1,2,3}$, Michael Spira $^{3}$ \\ ${ }^{1}$ Physik-Institut, Universität Zürich, 8057 Zurich, Switzerland \\ 2 Physics Department, ETH Zürich, 8093 Zurich, Switzerland \\ ${ }^{3}$ Paul Scherrer Institut PSI, 5232 Villigen, Switzerland
}

Received: 4 July 2018 / Accepted: 19 September 2018 / Published online: 4 October 2018

(C) The Author(s) 2018

\begin{abstract}
We consider Higgs boson production through gluon fusion at large transverse momentum in hadronic collisions. We present the analytic expressions of the relevant one-loop QCD amplitudes including the effects of the complete set of dimension-six operators. The latter correspond to modifications of the top and bottom Yukawa couplings, to an effective point-like Higgs coupling to gluons and to the chromomagnetic operator of the top quark. The quantitative impact of the chromomagnetic operator is also studied. Our results confirm previous findings that the effect of the chromomagnetic operator at high $p_{T}$ can be large and should not be neglected.
\end{abstract}

\section{Introduction}

After the discovery of the scalar resonance of mass $125 \mathrm{GeV}$ $[1,2]$ the measurement of its properties is one of the main activities of the LHC program. The Run I measurements [3] showed that the new resonance is compatible with the Standard Model Higgs boson. There is, however, still the possibility that more precise measurements will uncover small deviations from the Standard Model (SM) predictions. These might be the long lasting legacy of the LHC, which will encompass the searches for New Physics. The need of a consistent framework to capture small deviations from the SM is reflected in the wide discussions in Refs. [4-6]. The Standard Model Effective Field Theory (SMEFT) is a promising and theory motivated approach, in which the deviations from the SM are parametrised with higher-dimension operators, in the first approximation dimension six [7,8].

Next to the inclusive quantities, differential Higgs observables were measured in Run I [9-15] and with a partial data set of Run II [16-18], although still with relatively large

\footnotetext{
a e-mail: grazzini@physik.uzh.ch
}

uncertainties. With the increasing amount of collected data, the statistical accuracy will improve, thereby allowing us to put stringent constraints on the SMEFT parameters. One of the observables which is able to shed light on the structure of the Higgs sector is the transverse momentum spectrum $\left(p_{T}\right)$ of the Higgs particle. For example, a measurement of the $p_{T}$ spectrum could give insight on the nature of the Higgs boson coupling to gluons (see e.g. Refs. [19,20]).

Recent years have seen considerable progress in the theoretical control of the $p_{T}$ spectrum in the SM [21-27]. In particular, the NLO QCD corrections are now available including the exact dependence on the top-quark mass [28].

Dedicated calculations and tools are needed to enable the experimental analyses to set bounds on the SMEFT operators. Approximate results for the total gluon fusion Higgs production cross section including modified top and bottom Yukawa couplings and an additional direct $\mathrm{Hgg}$ interaction have been obtained at NNLO in QCD perturbation theory in Ref. [29] and at $\mathrm{N}^{3} \mathrm{LO}$ in Refs. [30,31]. As far as gluon fusion is concerned, the inclusion of dimension-six and dimension-eight operators in the Higgs $p_{T}$-spectrum also has been considered in Refs. [32-34] and [35,36], respectively. Strategies for extracting information on the Higgs-gluon couplings from the measurements were studied in Ref. [34], and the study the low- $p_{T}$ range therein was made possible by using Monte Carlo Parton Shower. Also in Ref. [20] the prospects of the determination of the Wilson coefficients in the high-luminosity LHC and future colliders were considered. The mentioned studies usually omitted the effects of the chromomagnetic operator, but a dedicated work analysed its effect on the LO Higgs production [37]. This was followed by a LO study [38] on the interplay of the SMEFT operators entering top-induced Higgs production channels, with the chromomagnetic operator treated in the heavy-top limit (HTL). Recently, the program of the SMEFT at NLO QCD was started by the MADGRAPH5_AMC@NLO group 
[39] and led to the calculation of $t \bar{t} H, t H$ [40] and recently also of Higgs production through gluon fusion [41].

In this work we recall the results for the LO Higgs production via gluon fusion and we extend our study [42] of the Higgs $p_{T}$ spectrum to include the effects of the chromomagnetic operator. More precisely, we present the analytic expressions of the relevant one-loop QCD amplitudes including the effects of the complete set of dimension-six operators and we shortly illustrate the impact of the chromomagnetic operator on the high- $p_{T}$ tail of the spectrum. Note that, due to the automated character of the calculations in the MADGRAPH5_AMC@NLO framework [40,41], they can be considered complementary to the analytic calculations presented here.

The paper is organised as follows. In Sect. 1 we review the LO results and set up our notation. In Sect. 2 we present the analytical results for the SMEFT one-loop QCD amplitudes in all partonic channels, and we briefly discuss the impact of the chromomagnetic operator at high $p_{T}$. In Sect. 4 we draw our conclusions.

\section{Framework and LO results}

We consider the effective Lagrangian

$\mathcal{L}=\mathcal{L}_{S M}+\sum_{i} \frac{c_{i}}{\Lambda^{2}} \mathcal{O}_{i}$

where the SM is supplemented by the inclusion of a set of dimension-six operators describing new physics effects at a scale $\Lambda$ well above the EW scale. We focus on the following three operators

$\mathcal{O}_{1}=|H|^{2} \bar{q}_{L} H^{c} t_{R}+$ h.c. $\quad \mathcal{O}_{2}=|H|^{2} G_{\mu \nu}^{a} G^{a, \mu \nu}$

$\mathcal{O}_{3}=\bar{Q}_{L} H \sigma^{\mu \nu} T^{a} t_{R} G_{\mu \nu}^{a}+$ h.c.

These operators, in the case of single Higgs production, may be rewritten as:

$\frac{c_{1}}{\Lambda^{2}} \mathcal{O}_{1} \rightarrow c_{1} \frac{m_{t}}{v} h \bar{t} t$

$\frac{c_{2}}{\Lambda^{2}} \mathcal{O}_{2} \rightarrow c_{2} \frac{\alpha_{\mathrm{S}}}{\pi v} h G_{\mu \nu}^{a} G^{a, \mu \nu}$,

$\frac{c_{3}}{\Lambda^{2}} \mathcal{O}_{3} \rightarrow c_{3} \frac{g_{\mathrm{S}} m_{t}}{2 v^{3}}(v+h) G_{\mu \nu}^{a}\left(\bar{t}_{L} \sigma^{\mu \nu} T^{a} t_{R}+\right.$ h.c $)$,

where $\alpha_{\mathrm{S}}$ is the QCD coupling $\left(\alpha_{\mathrm{S}}=g_{\mathrm{S}}^{2} /(4 \pi)\right), m_{t}$ is the (pole) mass of the top quark, $v$ is the expectation value of the Higgs field, $v=\left(\sqrt{2} G_{F}\right)^{-1 / 2} \sim 246 \mathrm{GeV}$ and $\sigma^{\mu \nu}=$ $\frac{i}{2}\left[\gamma^{\mu}, \gamma^{\nu}\right]$. The operator $\mathcal{O}_{1}$ is the Yukawa operator, and describes modifications of the $t \bar{t} H$ coupling. The operator $\mathcal{O}_{2}$ provides a contact interaction of the Higgs boson and gluons with the same structure as in the heavy-top limit of the SM. The operator $\mathcal{O}_{3}$ is the chromomagnetic dipole moment operator, which modifies the interactions between gluons and the top quark. In our convention, based on the SILH basis $[43,44]$, we express the Wilson coefficients as factors in the canonically normalized Lagrangian.

To set up our convention we reproduce the results for the LO inclusive cross section for $g g \rightarrow H$ as exemplified in Refs. $[42,45,46]$. After renormalizing the point-like Higgsgluon coupling $c_{2}$ in the $\overline{\mathrm{MS}}$ scheme the LO matrix element can be decomposed as

$$
\begin{aligned}
\mathcal{T}_{g g \rightarrow H}\left(p_{1}, p_{2}\right)= & i \frac{\alpha_{\mathrm{S}}}{3 \pi v} \epsilon_{\mu}\left(p_{1}\right) \epsilon_{v}\left(p_{2}\right) \\
& \times\left[p_{1}^{v} p_{2}^{\mu}-\left(p_{1} p_{2}\right) g^{\mu \nu}\right] F\left(\tau_{H}\right),
\end{aligned}
$$

where $p_{1}, p_{2}$ are the gluon momenta, $\epsilon\left(p_{1}\right), \epsilon\left(p_{2}\right)$ their polarisations and $\tau_{H}=4 m_{t}^{2} / m_{H}^{2}, m_{H}$ being the Higgs boson mass. The form factor $F(\tau)$ is defined as

$F(\tau)=c_{1} F_{1}(\tau)+c_{2}\left(\mu_{R}\right) F_{2}(\tau)+\operatorname{Re}\left(c_{3}\right) \frac{m_{t}^{2}}{v^{2}} F_{3}(\tau)$,

with $^{1}$

$F_{1}(\tau)=\frac{3}{2} \tau[1+(1-\tau) f(\tau)]$,

$F_{2}(\tau)=12$,

$F_{3}(\tau)=3\left(\tau f(\tau)+2 g(\tau)-1-2 \ln \frac{\mu_{R}^{2}}{m_{t}^{2}}\right)$

and the functions $f, g$ are defined in "Appendix". In the HTL the form factors approach the simple expressions

$F_{1}(\tau) \rightarrow 1$,

$F_{2}(\tau) \rightarrow 12$,

$F_{3}(\tau) \rightarrow 6\left(1-\ln \frac{\mu_{R}^{2}}{m_{t}^{2}}\right)$.

\section{Higgs plus jet production}

Higgs boson production in association with a jet is the LO contribution to Higgs boson production at finite transverse momenta. This process is mediated by $g g, g q$ and $q \bar{q}$ initial states. We start the presentation of our results for the $g g$ channel,

$g\left(p_{1}\right)+g\left(p_{2}\right) \rightarrow g\left(p_{3}\right)+H(q)$

and the Mandelstam variables are defined as

$$
\begin{array}{lc}
s=2 p_{1} \cdot p_{2} & t=-2 p_{1} \cdot p_{3} \\
u=-2 p_{2} \cdot p_{3} & \text { with } \quad s+t+u=m_{H}^{2} .
\end{array}
$$

${ }^{1}$ Note that we changed our sign convention of $c_{3}$ compared to Ref. [42]. 
Fig. 1 Generic diagrams originating from the $\mathcal{O}_{1}$ operator that also provide the SM contribution

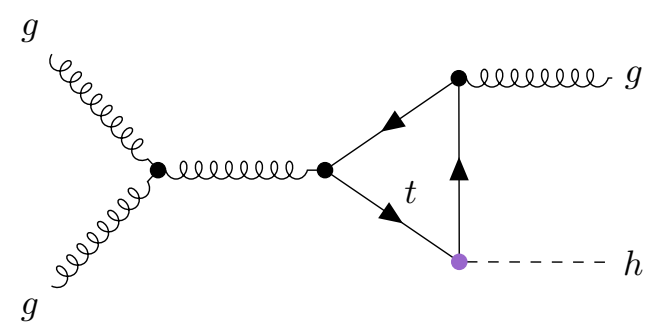

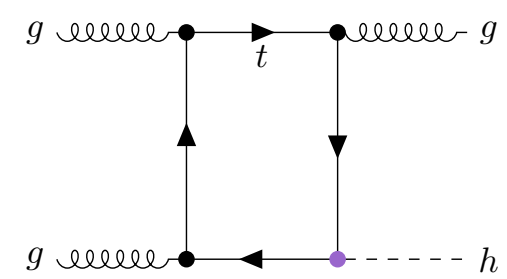

Fig. 2 Generic diagrams originating from the $\mathcal{O}_{2}$ operator. These correspond also to the effective SM diagrams in the HTL
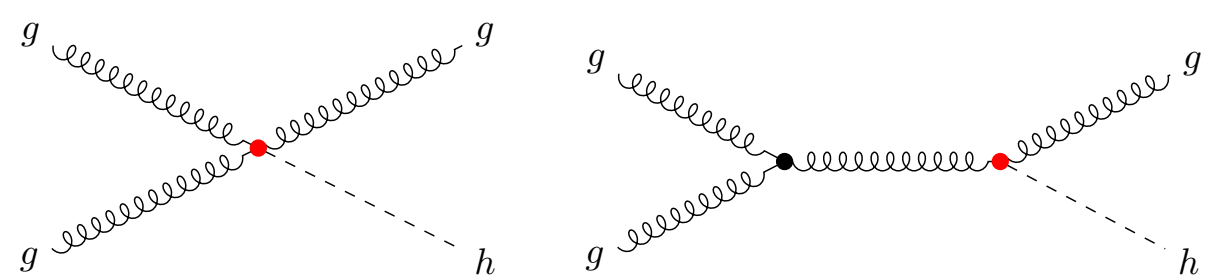
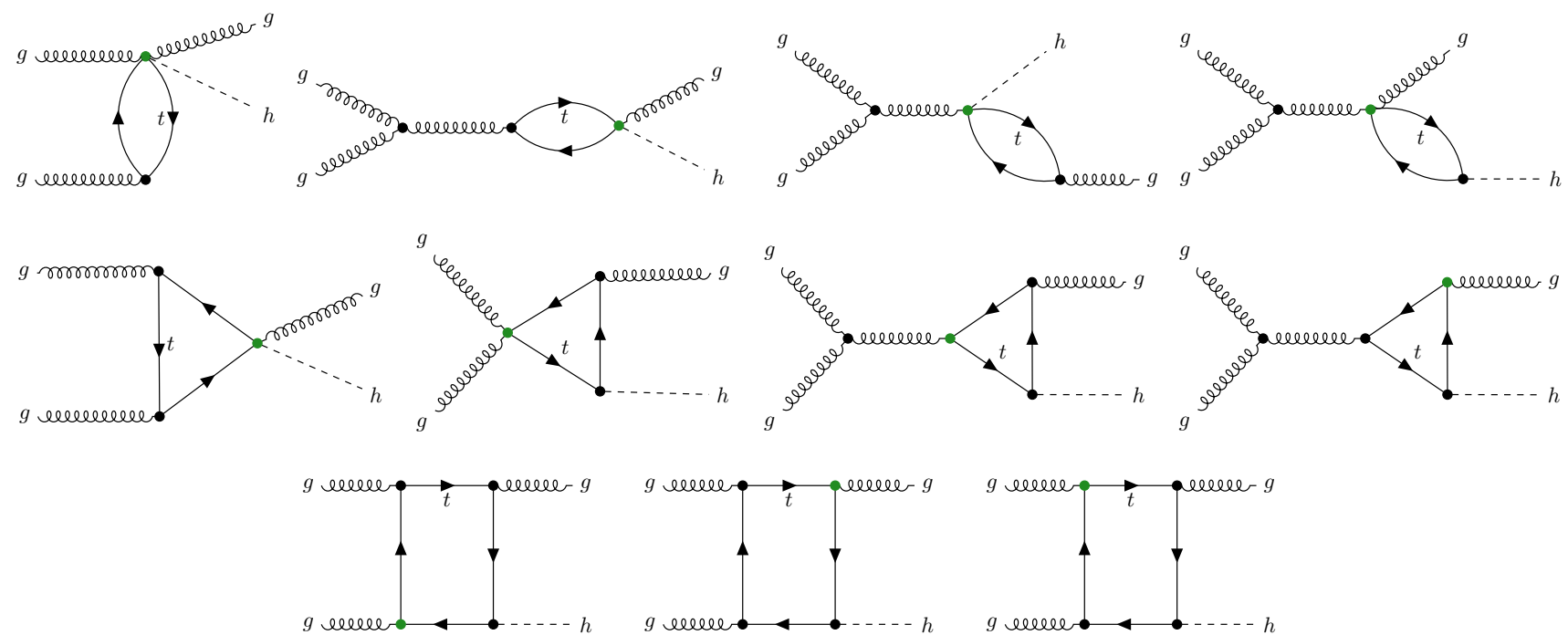

Fig. 3 Generic diagrams induced by the chromomagnetic operator $\mathcal{O}_{3}$

The contributing generic SM diagrams are shown in Fig. 1.

The contribution from the modified Yukawa coupling can be straightforwardly obtained by rescaling the SM result. The effective Higgs-gluon coupling gives rise to the diagrams in Fig. 2. When considering the insertion of the chromomagnetic operator we obtain 54 additional diagrams (see Fig. 3) out of which just 2 types are topologically equivalent to the SM ones.

The amplitude can be cast into the form

$$
\begin{aligned}
& \mathcal{T}_{g g \rightarrow g H}\left(p_{1}, p_{2}, p_{3}\right) \\
& \quad=\frac{i g_{S}^{3} m_{H}^{4}}{12 \pi^{2} v} f_{a b c} \mathcal{M}_{g g \rightarrow g H}^{\mu v \rho} \epsilon_{\mu}\left(p_{1}\right) \epsilon_{v}\left(p_{2}\right) \epsilon_{\rho}^{*}\left(p_{3}\right),
\end{aligned}
$$

where $\epsilon\left(p_{i}\right)$ are the gluon polarisation vectors. The amplitude can be decomposed into four independent tensor structures [47]

$$
\begin{aligned}
& \mathcal{M}_{g g \rightarrow g H}^{\mu \nu \rho}\left(p_{1}, p_{2}, p_{3}\right)=F_{1}\left(p_{1}, p_{2}, p_{3}\right) \mathcal{Q}_{1}^{\mu \nu \rho} \\
& \quad+F_{2}\left(p_{1}, p_{2}, p_{3}\right) \mathcal{Q}_{2}^{\mu \nu \rho} \\
& \quad+F_{3}\left(p_{1}, p_{2}, p_{3}\right) \mathcal{Q}_{3}^{\mu \nu \rho}+F_{4}\left(p_{1}, p_{2}, p_{3}\right) \mathcal{Q}_{4}^{\mu \nu \rho}
\end{aligned}
$$

where

$$
\begin{aligned}
\mathcal{Q}_{1}^{\mu \nu \rho}= & p_{1}^{\rho} p_{2}^{\mu} p_{3}^{\nu}-p_{1}^{\nu} p_{2}^{\rho} p_{3}^{\mu} \\
& +g^{\mu \nu}\left[\left(p_{1} \cdot p_{3}\right) p_{2}^{\rho}-\left(p_{2} \cdot p_{3}\right) p_{1}^{\rho}\right] \\
& +g^{\mu \rho}\left[\left(p_{2} \cdot p_{3}\right) p_{1}^{\nu}-\left(p_{1} \cdot p_{2}\right) p_{3}^{\nu}\right] \\
& +g^{\nu \rho}\left[\left(p_{1} \cdot p_{2}\right) p_{3}^{\mu}-\left(p_{1} \cdot p_{3}\right) p_{2}^{\mu}\right] \\
\mathcal{Q}_{2}^{\mu \nu \rho}= & {\left[\left(p_{2} \cdot p_{3}\right) p_{1}^{\rho}-\left(p_{1} \cdot p_{3}\right) p_{2}^{\rho}\right] \frac{p_{1}^{v} p_{2}^{\mu}-\left(p_{1} \cdot p_{2}\right) g^{\mu \nu}}{\left(p_{1} \cdot p_{2}\right)} }
\end{aligned}
$$




$$
\begin{aligned}
& \mathcal{Q}_{3}^{\mu \nu \rho}=\left[\left(p_{2} \cdot p_{3}\right) p_{1}^{\nu}-\left(p_{1} \cdot p_{2}\right) p_{3}^{\nu}\right] \frac{p_{1}^{\rho} p_{3}^{\mu}-\left(p_{1} \cdot p_{3}\right) g^{\mu \rho}}{\left(p_{1} \cdot p_{3}\right)} \\
& \mathcal{Q}_{4}^{\mu \nu \rho}=\left[\left(p_{1} \cdot p_{3}\right) p_{2}^{\mu}-\left(p_{1} \cdot p_{2}\right) p_{3}^{\mu}\right] \frac{p_{2}^{\rho} p_{3}^{\nu}-\left(p_{2} \cdot p_{3}\right) g^{\nu \rho}}{\left(p_{2} \cdot p_{3}\right)} .
\end{aligned}
$$

It is useful to note that based on the definitions of the tensor structures the form factors have the following properties due to Bose symmetry,

$$
\begin{aligned}
F_{2}\left(p_{1}, p_{2}, p_{3}\right) & =F_{2}\left(p_{2}, p_{1}, p_{3}\right) \\
& =-F_{3}\left(p_{1}, p_{3}, p_{2}\right)=F_{4}\left(p_{3}, p_{2}, p_{1}\right)
\end{aligned}
$$

and $F_{1}$ is totally symmetric. When squaring the amplitude, the decomposition in Eq. (16) leads to mixed terms in the form factors $F_{i}$. If we rearrange the form factors as

$$
\begin{aligned}
C_{1}\left(s, t, u ; m_{t}\right)= & \frac{s t u}{2}\left[2 F_{1}\left(p_{1}, p_{2}, p_{3}\right)+F_{2}\left(p_{1}, p_{2}, p_{3}\right)\right. \\
& \left.-F_{3}\left(p_{1}, p_{2}, p_{3}\right)+F_{4}\left(p_{1}, p_{2}, p_{3}\right)\right] \\
C_{2}\left(s, t, u ; m_{t}\right)= & \frac{s t u}{2} F_{2}\left(p_{1}, p_{2}, p_{3}\right) \\
C_{3}\left(s, t, u ; m_{t}\right)= & \frac{s t u}{2} F_{3}\left(p_{1}, p_{2}, p_{3}\right) \\
C_{4}\left(s, t, u ; m_{t}\right)= & \frac{s t u}{2} F_{4}\left(p_{1}, p_{2}, p_{3}\right)
\end{aligned}
$$

where the $C_{i}$ develop the analogous Bose-symmetry properties as the previous form factors

$$
\begin{aligned}
& C_{3}\left(s, t, u ; m_{t}\right)=-C_{2}\left(t, s, u ; m_{t}\right) \\
& C_{4}\left(s, t, u ; m_{t}\right)=C_{2}\left(u, t, s ; m_{t}\right)
\end{aligned}
$$

the amplitude squared can now be expressed as a sum of absolute squares

$$
\left|\mathcal{T}_{g g \rightarrow g H}\right|^{2}=\frac{32 \alpha_{S}^{3}}{3 \pi v^{2}} \frac{m_{H}^{8}}{s t u}\left(\left|C_{1}\right|^{2}+\left|C_{2}\right|^{2}+\left|C_{3}\right|^{2}+\left|C_{4}\right|^{2}\right)
$$

Here we present the results for $C_{1}\left(s, t, u ; m_{t}\right)$ and $C_{2}(s, t$, $\left.u ; m_{t}\right)\left(C_{3}\right.$ and $C_{4}$ can be obtained from $C_{2}$ following Eq. (20)) in terms of the scalar integrals:

$C_{i}^{(\alpha)}=\frac{1}{32} \tau_{H}^{2} \sum_{j=1}^{12} P_{i, j}^{(\alpha)} T_{j}$

where the $\alpha$ index runs over the operators $\mathcal{O}_{\alpha}$ in Eq. (2) and

$$
T_{1}=1 \quad T_{2}=2\left[1-g\left(\tau_{s}\right)\right]
$$

$$
\begin{aligned}
T_{3} & =2\left[1-g\left(\tau_{t}\right)\right] & T_{4} & =2\left[1-g\left(\tau_{u}\right)\right] \\
T_{5} & =2\left[1-g\left(\tau_{H}\right)\right] & T_{6} & =2 f\left(\tau_{s}\right) \\
T_{7} & =2 f\left(\tau_{t}\right) & T_{8} & =2 f\left(\tau_{u}\right) \\
T_{9} & =2 f\left(\tau_{H}\right) & T_{10} & =J(\tilde{s}, \tilde{t}, \tilde{u}) \\
T_{11} & =J(\tilde{s}, \tilde{u}, \tilde{t}) & T_{12} & =J(\tilde{u}, \tilde{s}, \tilde{t}) .
\end{aligned}
$$

In order to present our results in a compact form we have defined dimensionless quantities $\tilde{x}=x / m_{t}^{2}$ and $\tau_{x}=4 / \tilde{x}$ for $x=s, t, u$ and $\rho=m_{H}^{2} / m_{t}^{2}$. The scalar functions $f(x)$, $g(x)$ and $J(x, y, z)$ are given in "Appendix". The $P_{i, j}^{(1)}$ coefficients, corresponding to the SM contribution, read

$$
\begin{aligned}
& P_{1,1}^{(1)}=12 \rho \quad P_{1,2}^{(1)}=0 \\
& P_{1,3}^{(1)}=0 \quad P_{1,4}^{(1)}=0 \\
& P_{1,5}^{(1)}=0 \quad P_{1,6}^{(1)}=3(4-\rho) \\
& P_{1,7}^{(1)}=3(4-\rho) \quad P_{1,8}^{(1)}=3(4-\rho) \\
& P_{1,9}^{(1)}=-9(4-\rho) \quad P_{1,10}^{(1)}=\frac{3}{2} \tilde{s} \tilde{u}(4-\rho) \\
& P_{1,11}^{(1)}=\frac{3}{2} \tilde{s} \tilde{t}(4-\rho) P_{1,12}^{(1)}=\frac{3}{2} \tilde{t} \tilde{u}(4-\rho) \\
& P_{2,1}^{(1)}=-12 \tilde{s} \frac{\tilde{u} \tilde{t}-\tilde{s}^{2}}{(\tilde{s}+\tilde{u})(\tilde{s}+\tilde{t})} \\
& P_{2,2}^{(1)}=0 \\
& P_{2,3}^{(1)}=\frac{12 \tilde{t} \tilde{u}(\tilde{u}+2 \tilde{s})}{(\tilde{s}+\tilde{u})^{2}} \\
& P_{2,4}^{(1)}=\frac{12 \tilde{t} \tilde{u}(\tilde{t}+2 \tilde{s})}{(\tilde{s}+\tilde{t})^{2}} \\
& P_{2,5}^{(1)}=-\frac{12 \tilde{t} \tilde{u}}{(\tilde{s}+\tilde{t})^{2}(\tilde{s}+\tilde{u})^{2}}\left[\left(3 \tilde{s}^{2}+\tilde{t} \tilde{u}+2 \rho \tilde{s}\right)(\rho-\tilde{s})+4 \tilde{s}^{3}\right] \\
& P_{2,6}^{(1)}=-3(\tilde{s}-4) \\
& P_{2,7}^{(1)}=-\frac{3\left(4 \tilde{t} \tilde{u}(\tilde{s}+\tilde{u})^{2}+\tilde{s}(4-\tilde{s})\left(\tilde{u}^{2}-\tilde{s}^{2}\right)+8 \tilde{s}^{2} \tilde{u}\right)}{\tilde{s}(\tilde{s}+\tilde{u})^{2}} \\
& P_{2,8}^{(1)}=-\frac{3\left(4 \tilde{t} \tilde{u}(\tilde{s}+\tilde{t})^{2}+\tilde{s}(4-\tilde{s})\left(\tilde{t}^{2}-\tilde{s}^{2}\right)+8 \tilde{s}^{2} \tilde{t}\right)}{\tilde{s}(\tilde{s}+\tilde{t})^{2}} \\
& P_{2,9}^{(1)}=\frac{3}{\tilde{s}(\tilde{s}+\tilde{t})^{2}(\tilde{s}+\tilde{u})^{2}}\left\{4 \tilde{u}(\tilde{s}+\tilde{t})^{2}\left[2 \tilde{s}^{2}+\tilde{t}(\tilde{s}+\tilde{u})^{2}\right]\right. \\
& +8 \tilde{s}^{4} \tilde{t}+4 \tilde{s}^{2} \tilde{t} \tilde{u}\left(\tilde{s}^{2}+2 \tilde{u}\right) \\
& \left.+\tilde{s}(\tilde{s}-4)\left[\tilde{s}^{2}\left[(\tilde{s}+\tilde{t})^{2}+(\tilde{s}+\tilde{u})^{2}\right]+\tilde{s}^{3}(2 \rho-\tilde{s})-\tilde{t}^{2} \tilde{u}^{2}\right]\right\} \\
& P_{2,10}^{(1)}=\frac{3 \tilde{s} \tilde{u}(4-\tilde{s})}{2} \\
& P_{2,11}^{(1)}=\frac{3 \tilde{s} \tilde{t}(4-\tilde{s})}{2} \\
& P_{2,12}^{(1)}=-\frac{3 \tilde{t} \tilde{u}\left(4 \tilde{t} \tilde{u}-\tilde{s}^{2}+12 \tilde{s}\right)}{2 \tilde{s}} .
\end{aligned}
$$

This result agrees with the one presented in Refs. [47-49]. 
The coefficients for the contribution arising from the chromomagnetic operator read

$$
\begin{aligned}
& P_{1,1}^{(3)}=6 \rho^{2}\left(1-2 \log \frac{\mu_{R}^{2}}{m_{t}^{2}}\right) P_{1,2}^{(3)}=-6 \tilde{t} \tilde{u} \\
& P_{1,3}^{(3)}=-6 \tilde{s} \tilde{u} \quad P_{1,4}^{(3)}=-6 \tilde{s} \tilde{t} \\
& P_{1,5}^{(3)}=-6 \rho^{2} \quad P_{1,6}^{(3)}=-3(2 \rho-\tilde{t} \tilde{u}) \\
& P_{1,7}^{(3)}=-3(2 \rho-\tilde{s} \tilde{u}) \quad P_{1,8}^{(3)}=-3(2 \rho-\tilde{s} \tilde{t}) \\
& P_{1,9}^{(3)}=18 \rho \quad P_{1,10}^{(3)}=-3 \tilde{s} \tilde{u}(\rho+\tilde{t}) \\
& P_{1,11}^{(3)}=-3 \tilde{s} \tilde{t}(\rho+\tilde{u}) \quad P_{1,12}^{(3)}=-3 \tilde{t} \tilde{u}(\rho+\tilde{s}) \\
& P_{2,1}^{(3)}=\frac{6 \rho \tilde{s}\left(\tilde{s}^{2}-\tilde{t} \tilde{u}\right)}{(\tilde{s}+\tilde{t})(\tilde{s}+\tilde{u})}-12 \tilde{s}^{2} \log \frac{\mu_{R}^{2}}{m_{t}^{2}} \\
& P_{2,2}^{(3)}=0 \\
& P_{2,3}^{(3)}=\frac{6 \tilde{t} \tilde{u}\left(\rho^{2}-\tilde{t}(\tilde{t}+\tilde{u})\right)}{(\tilde{s}+\tilde{u})^{2}} \\
& P_{2,4}^{(3)}=\frac{6 \tilde{t} \tilde{u}\left(\rho^{2}-\tilde{u}(\tilde{t}+\tilde{u})\right)}{(\tilde{s}+\tilde{t})^{2}} \\
& P_{2,5}^{(3)}=-\frac{6 \rho}{(\tilde{s}+\tilde{t})^{2}(\tilde{s}+\tilde{u})^{2}}\left(\rho \tilde{s}^{2}\left(\tilde{s}^{2}+4 \tilde{t} \tilde{u}\right)\right. \\
& \left.+2 \tilde{s} \tilde{t} \tilde{u}(\rho-\tilde{s})^{2}-\tilde{t}^{2} \tilde{u}^{2}(2 \tilde{s}-\rho)\right) \\
& P_{2,6}^{(3)}=-6(2 \tilde{s}-\rho) \\
& P_{2,7}^{(3)}=-\frac{3\left\{(\tilde{s}+\tilde{u})^{2}[2 \rho(\tilde{s}+\tilde{t} \tilde{u})-\tilde{s} \tilde{t} \tilde{u}]-4 \tilde{s}^{2}\left[\tilde{s}(\rho-\tilde{s})+\tilde{u}^{2}\right]\right\}}{\tilde{s}(\tilde{s}+\tilde{u})^{2}} \\
& P_{2,8}^{(3)}=-\frac{3\left\{(\tilde{s}+\tilde{t})^{2}[2 \rho(\tilde{s}+\tilde{t} \tilde{u})-\tilde{s} \tilde{t} \tilde{u}]-4 \tilde{s}^{2}\left[\tilde{s}(\rho-\tilde{s})+\tilde{t}^{2}\right]\right\}}{\tilde{s}(\tilde{s}+\tilde{t})^{2}} \\
& P_{2,9}^{(3)}=\frac{3}{\tilde{s}(\tilde{s}+\tilde{t})^{2}(\tilde{s}+\tilde{u})^{2}} \\
& \times\left\{2 \tilde { s } \left[3 \rho \tilde{s}^{4}+\tilde{s}(2 \tilde{t} \tilde{u}-\rho \tilde{s})(\rho-\tilde{s})^{2}+2 \tilde{s}^{2} \tilde{t} \tilde{u}(\tilde{s}+4 \rho)\right.\right. \\
& \left.\left.+\tilde{t}^{2} \tilde{u}^{2}(\rho-2 \tilde{s})\right]+\tilde{t} \tilde{u}(\tilde{s}+\tilde{t})^{2}(\tilde{s}+\tilde{u})^{2}(2 \rho-\tilde{s})\right\} \\
& P_{2,10}^{(3)}=-3 \tilde{s} \tilde{u}(\tilde{s}-\tilde{u}) \\
& P_{2,11}^{(3)}=-3 \tilde{s} \tilde{t}(\tilde{s}-\tilde{t}) \\
& P_{2,12}^{(3)}=-\frac{3 \tilde{t} \tilde{u}[2(\rho-\tilde{s})(3 \tilde{s}+\tilde{t} \tilde{u})+\tilde{s} \tilde{t} \tilde{u}]}{2 \tilde{s}} .
\end{aligned}
$$

The $\log \frac{\mu_{R}^{2}}{m_{t}^{2}}$ terms arise from the absorption of the $1 / \epsilon$ divergence in the renormalisation of the $c_{g}$ coupling. For the effective Higgs coupling to gluons we directly present the expressions of the $C_{1}$ and $C_{2}$ form factors, which read

$$
\begin{aligned}
& C_{1}^{(2)}\left(s, t, u ; m_{t}\right)=12 \\
& C_{2}^{(2)}\left(s, t, u ; m_{t}\right)=\frac{12 s^{2}}{m_{H}^{4}},
\end{aligned}
$$

which correspond also to the HTL of the SM result multiplied by a factor of 12 . For completeness we report the HTL also for the contribution of the chromomagnetic operator

$$
C_{i}^{(3)} \rightarrow \frac{1}{2}\left(1-\ln \frac{\mu_{R}^{2}}{m_{t}^{2}}\right) C_{i}^{(2)} \quad i=1,2 .
$$

The final results for the form factors $C_{i}$ read

$$
\begin{aligned}
& C_{i}\left(s, t, u ; m_{t}\right)=c_{1} C_{i}^{(1)}\left(s, t, u ; m_{t}\right) \\
& \quad+c_{2}\left(\mu_{R}\right) C_{i}^{(2)}\left(s, t, u ; m_{t}\right)+\operatorname{Re}\left(c_{3}\right) \frac{m_{t}^{2}}{v^{2}} C_{i}^{(3)}\left(s, t, u ; m_{t}\right) .
\end{aligned}
$$

We now move to the $q \bar{q}$ channel:

$q\left(p_{1}\right)+\bar{q}\left(p_{2}\right) \rightarrow g\left(p_{3}\right)+H(q)$.

The contributing generic Feynman diagrams are depicted in Fig. 4.

The corresponding amplitude can be decomposed as

$$
\begin{aligned}
& \mathcal{M}_{q \bar{q} \rightarrow g H}\left(p_{1}, p_{2}, p_{3}\right)=\frac{i g_{S}^{3}}{16 \pi^{2} v s} \bar{v}\left(p_{2}\right) \gamma^{\mu} T^{a} u\left(p_{1}\right) \\
& \quad \times\left[g_{\mu \nu}\left(p_{1}+p_{2}\right) \cdot p_{3}-p_{3 \mu}\left(p_{1}+p_{2}\right)_{\nu}\right] \\
& \epsilon^{\nu}\left(p_{3}\right) D\left(p_{1}, p_{2}, p_{3}\right) .
\end{aligned}
$$

We again present the results in the form

$$
D^{(\alpha)}\left(p_{1}, p_{2}, p_{3}\right)=\sum_{j=1}^{5} P_{j}^{(\alpha)} T_{j} \quad \alpha=1,2,3
$$

with the basis of the scalar integrals:

$$
\begin{aligned}
& T_{1}=1 \\
& T_{2}=2\left[1-g\left(\tau_{s}\right)\right] T_{3}=2\left[1-g\left(\tau_{H}\right)\right] \\
& T_{4}=2 f\left(\tau_{s}\right) \quad T_{5}=2 f\left(\tau_{H}\right) .
\end{aligned}
$$

The coefficients corresponding to the SM and the Yukawa modifying operator in the SMEFT case read:

$$
\begin{aligned}
& P_{1}^{(1)}=\frac{8}{(\tilde{s}-\rho)} \\
& P_{2}^{(1)}=\frac{8 \tilde{s}}{(\tilde{s}-\rho)^{2}} \\
& P_{3}^{(1)}=-\frac{8 \tilde{s}}{(\tilde{s}-\rho)^{2}} \\
& P_{4}^{(1)}=-\frac{4(4+\tilde{s}-\rho)}{(\tilde{s}-\rho)^{2}} \\
& P_{5}^{(1)}=\frac{4(4+\tilde{s}-\rho)}{(\tilde{s}-\rho)^{2}} .
\end{aligned}
$$

The contribution of the chromomagnetic operator reads:

$$
\begin{aligned}
& P_{1}^{(3)}=\frac{4\left[\rho+2(\tilde{s}-\rho) \ln \frac{\mu_{R}^{2}}{m_{t}^{2}}\right]}{(\tilde{s}-\rho)} \\
& P_{2}^{(3)}=\frac{2\left[2 \tilde{s}^{2}+(\tilde{s}-\rho)^{2}\right]}{(\tilde{s}-\rho)^{2}}
\end{aligned}
$$



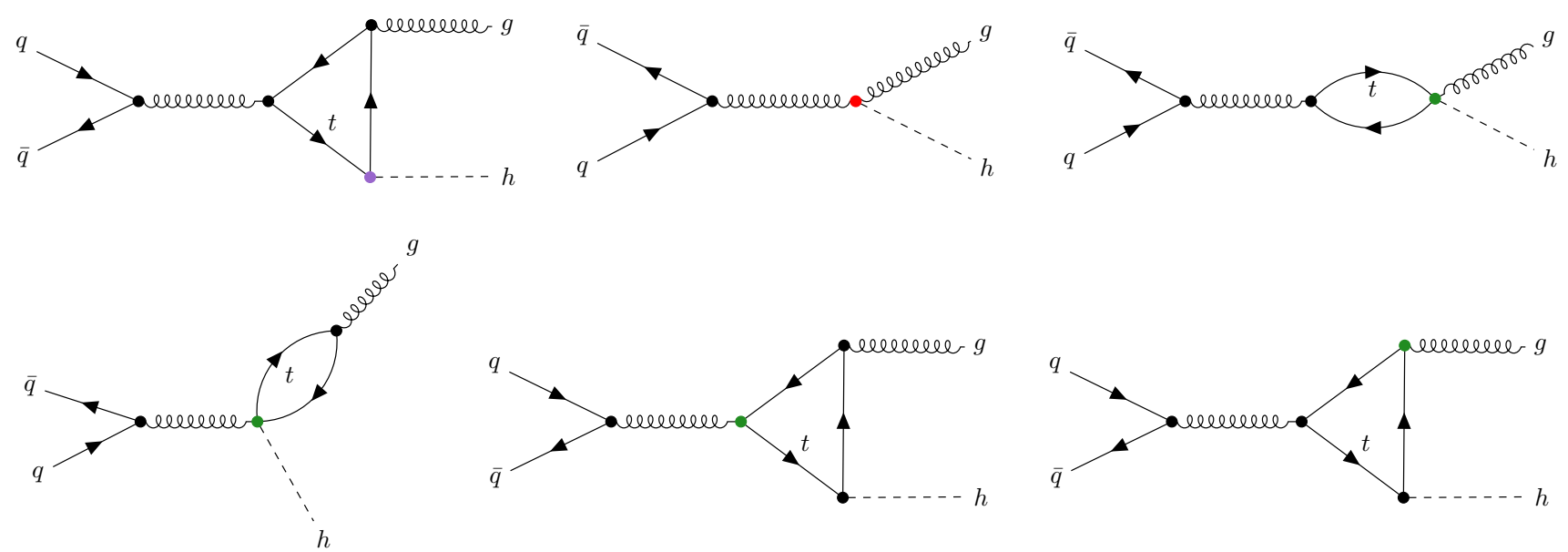

Fig. 4 Generic diagrams of the $q \bar{q}$-channel diagrams in the SMEFT. The color coding is the same as in Figs. 1-3

$P_{3}^{(3)}=-\frac{4 \rho(2 \tilde{s}-\rho)}{(\tilde{s}-\rho)^{2}}$

$P_{4}^{(3)}=-\frac{8(2 \tilde{s}-\rho)}{(\tilde{s}-\rho)^{2}}$

$P_{5}^{(3)}=\frac{8(2 \tilde{s}-\rho)}{(\tilde{s}-\rho)^{2}}$.

Again, we finalise the results by presenting the amplitude for the point-like Higgs coupling to gluons which corresponds to the HTL of the SM:

$D^{(2)}\left(p_{1}, p_{2}, p_{3}\right)=-16$.

The HTL of the relevant operators reads:

$D^{(1)} \rightarrow \frac{1}{12} D^{(2)}=-\frac{4}{3}$

$D^{(3)} \rightarrow \frac{1}{2}\left(1-\ln \frac{\mu_{R}^{2}}{m_{t}^{2}}\right) D^{(2)}=-8\left(1-\ln \frac{\mu_{R}^{2}}{m_{t}^{2}}\right)$.

The final expression for the form factor is given by

$$
\begin{aligned}
& D\left(p_{1}, p_{2}, p_{3}\right) \\
& =c_{1} D^{(1)}\left(p_{1}, p_{2}, p_{3}\right)+c_{2}\left(\mu_{R}\right) D^{(2)}\left(p_{1}, p_{2}, p_{3}\right) \\
& \quad+\operatorname{Re}\left(c_{3}\right) \frac{m_{t}^{2}}{v^{2}} D^{(3)}\left(p_{1}, p_{2}, p_{3}\right) .
\end{aligned}
$$

The result for the $q g$ channel can be obtained by crossing.

The above results allow us to obtain complete predictions for Higgs boson production at high $p_{T}$ in the SMEFT. In Ref. [42] the effects of the $\mathcal{O}_{1}$ and $\mathcal{O}_{2}$ were studied, including the resummation of the large logarithmic contributions at small $p_{T}$, but neglecting the contribution of the chromomagnetic operator. We thus focus here on the effect of the chromomagnetic operator at high $p_{T}$. We consider $p p$ collisions at $\sqrt{s}=13 \mathrm{TeV}$ and use PDF4LHC2015 NLO

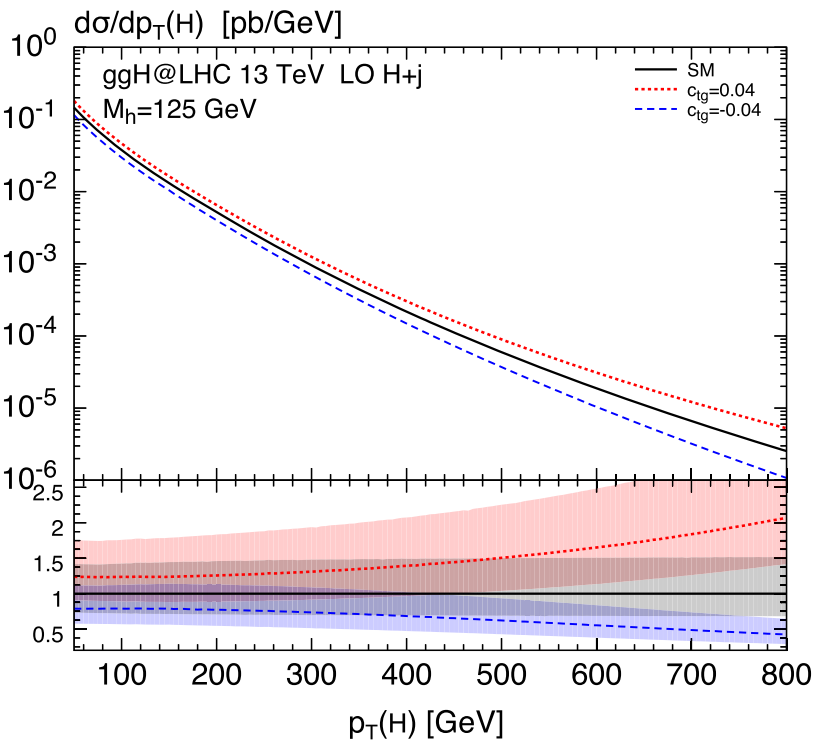

Fig. 5 Impact of the chromomagnetic operator on the $p_{T}$ spectrum of the Higgs boson in the region allowed by the current experimental constraints

parton distributions [50-55]. The central value of the renormalization and factorization scales is fixed to $\mu_{F}=\mu_{R}=$ $0.5 \times \sqrt{m_{H}^{2}+p_{T}^{2}}$.

In Fig. 5 we show the impact of the operator $\mathcal{O}_{3}$, by considering a variation of the coefficient $c_{3}$ within the range suggested by the study of Ref. [56]. The $p_{T}$ spectrum including the impact of the chromomagnetic operator is normalised to the SM result. Perturbative uncertainties are estimated with the usual 7-point scale variations. The numerical results, obtained with a modified version of the program HIGLU [57], show that the chromomagnetic operator can significantly affect the $p_{T}$ spectrum, and the effects start to exceed the scale uncertainty of the SM result around $p_{T} \approx 400$ $500 \mathrm{GeV}$. Considering also the improvements in the theoreti- 
cal control of the SM prediction [21-23,28], this means that at large transverse momenta the effect of the chromomagnetic operator could be clearly discriminated with respect to the SM prediction. Our numerical results agree with those of Ref. [40]. A more detailed study will be presented elsewhere.

\section{Conclusions}

In this work we studied Higgs boson production at large transverse momentum in gluon fusion within the SMEFT. We provided explicit and compact analytical results for the one-loop matrix elements of the corresponding partonic processes $g g, q \bar{q} \rightarrow H g$ and $g q \rightarrow H q$. The results, which are expressed in terms of standard one-loop scalar integrals, require the renormalization of the dimension-six point-like Higgs coupling to gluons in accordance with the related renormalization of the inclusive cross section $[38,42]$. We studied the quantitative impact of the chromomagnetic operator and found that it can significantly distort the transverse-momentum spectrum of the Higgs boson at large $p_{T}$. Depending on the actual size of the corresponding Wilson coefficient, this contribution has to be taken into account for a solid study of relevant dimension-six operators within the SMEFT. Turning this argument around, the Higgs transverse-momentum distribution will provide a relevant observable to constrain the Wilson coefficient of the chromomagnetic operator when significant statistics is accumulated.

Acknowledgements We would like to thank Fabio Maltoni and Eleni Vryonidou for useful correspondence on the results of Ref. [40]. This research was supported in part by the Swiss National Science Foundation (SNF) under contracts CRSII2-141847, 200020-169041 and by the Research Executive Agency (REA) of the European Union under the Grant Agreement number PITN-GA-2012-316704 (HiggsTools).

Open Access This article is distributed under the terms of the Creative Commons Attribution 4.0 International License (http://creativecomm ons.org/licenses/by/4.0/), which permits unrestricted use, distribution, and reproduction in any medium, provided you give appropriate credit to the original author(s) and the source, provide a link to the Creative Commons license, and indicate if changes were made.

Funded by SCOAP ${ }^{3}$.

\section{Appendix: Scalar integrals}

In this appendix we present the definitions of the functions $f, g, J$ used for our analytical results and their relation to the scalar one-loop integrals.

$$
g(\tau)= \begin{cases}\sqrt{\tau-1} \arcsin \frac{1}{\sqrt{\tau}} & \tau \geq 1 \\ \frac{\sqrt{1-\tau}}{2}\left[\ln \frac{1+\sqrt{1-\tau}}{1-\sqrt{1-\tau}}-i \pi\right] & \tau<1 .\end{cases}
$$

$$
\begin{aligned}
& f(\tau)= \begin{cases}\arcsin ^{2} \frac{1}{\sqrt{\tau}} & \tau \geq 1 \\
-\frac{1}{4}\left[\ln \frac{1+\sqrt{1-\tau}}{1-\sqrt{1-\tau}}-i \pi\right]^{2} & \tau<1\end{cases} \\
& J(\tilde{s}, \tilde{t}, \tilde{u})=I_{3}(\tilde{s}, \tilde{t}, \tilde{u}, \tilde{s})+I_{3}(\tilde{s}, \tilde{t}, \tilde{u}, \tilde{u})-I_{3}(\tilde{s}, \tilde{t}, \tilde{u}, \rho) \\
& I_{3}(\tilde{s}, \tilde{t}, \tilde{u}, \tilde{x})=\frac{1}{\tilde{s} \tilde{u}} \frac{2}{\beta_{+}-\beta_{-}}\left(\operatorname{Li}_{2}\left(\frac{\beta_{-}}{\beta_{-}-\alpha_{-}}\right)\right. \\
& -\mathrm{Li}_{2}\left(\frac{\beta_{+}}{\beta_{+}-\alpha_{+}}\right)+\mathrm{Li}_{2}\left(\frac{\beta_{-}}{\beta_{-}-\alpha_{+}}\right) \\
& \left.-\mathrm{Li}_{2}\left(\frac{\beta_{+}}{\beta_{+}-\alpha_{-}}\right)+\log \left(-\frac{\beta_{+}}{\beta_{-}}\right) \log \left(1+\frac{\tilde{x} \tilde{t}}{\tilde{s} \tilde{u}}\right)\right),
\end{aligned}
$$

with $\alpha_{ \pm}=\frac{1}{2}\left(1 \pm \sqrt{1-\frac{4}{\tilde{x}}}\right)$ and $\beta_{ \pm}=\frac{1}{2}\left(1 \pm \sqrt{1-\frac{4 \tilde{\tilde{T}}}{\tilde{\tilde{u}}}}\right)$. The function $\mathrm{Li}_{2}$ denotes the Spence function. The functions $f, g, J$ are related to the corresponding scalar one-loop integrals as $(n=4-2 \epsilon)$

$$
\begin{aligned}
& B_{0}\left(p ; m_{t}, m_{t}\right)=\int \frac{d^{n} k}{(2 \pi)^{n}} \frac{\mu^{2 \epsilon}}{\left(k^{2}-m_{t}^{2}\right)\left[(k+p)^{2}-m_{t}^{2}\right]} \\
&= i \frac{\Gamma(1+\epsilon)}{(4 \pi)^{2}}\left(\frac{4 \pi \mu^{2}}{m_{t}^{2}}\right)^{\epsilon}\left[\frac{1}{\epsilon}+2-2 g(\tau)\right]+\mathcal{O}(\epsilon) \\
&\left(\tau=4 m_{t}^{2} / p^{2}\right) \\
& C_{0}\left(p_{1}, p_{2} ; m_{t}, m_{t}, m_{t}\right) \int \frac{d^{n} k}{(2 \pi)^{n}} \frac{\mu^{2 \epsilon}}{\left(k^{2}-m_{t}^{2}\right)\left[\left(k-p_{1}\right)^{2}-m_{t}^{2}\right]\left[\left(k+p_{2}\right)^{2}-m_{t}^{2}\right]} \\
&= \frac{i}{(4 \pi)^{2} m_{t}^{2}}\left[-\frac{\tau}{2} f(\tau)\right]+\mathcal{O}(\epsilon) \quad\left(\tau=4 m_{t}^{2} /\left(2 p_{1} p_{2}\right)\right) \\
& D_{0}\left(p_{1}, p_{2}, p_{3} ; m_{t}, m_{t}, m_{t}, m_{t}\right) \\
&=\int \frac{d^{n} k}{(2 \pi)^{n}} \\
& \quad \times \frac{\mu^{2 \epsilon}}{\left(k^{2}-m_{t}^{2}\right)\left[\left(k+p_{1}\right)^{2}-m_{t}^{2}\right]\left[\left(k+p_{12}\right)^{2}-m_{t}^{2}\right]\left[\left(k+p_{123}\right)^{2}-m_{t}^{2}\right]} \\
&=\frac{i}{(4 \pi)^{2} m_{t}^{4}} J\left(\frac{s}{m_{t}^{2}}, \frac{t}{m_{t}^{2}}, \frac{u}{m_{t}^{2}}\right)+\mathcal{O}(\epsilon)
\end{aligned}
$$

where $p_{12}=p_{1}+p_{2}, p_{123}=p_{1}+p_{2}+p_{3}$ and $s=$ $2 p_{1} p_{2}, t=2 p_{1} p_{3}, u=2 p_{2} p_{3}$ where all $p_{i}(i=1,2,3)$ correspond to incoming massless external particles $\left(p_{i}^{2}=0\right)$.

\section{References}

1. ATLAS Collaboration, G. Aad et al., Observation of a new particle in the search for the Standard Model Higgs boson with the ATLAS detector at the LHC, Phys. Lett. B 716, 1-29 (2013). arXiv:1207.7214

2. CMS Collaboration, S. Chatrchyan et al., Observation of a new boson at a mass of $125 \mathrm{GeV}$ with the CMS experiment at the LHC. Phys. Lett. B 716, 30-61 (2013). arXiv:1207.7235

3. ATLAS, CMS Collaboration, G. Aad et al., Measurements of the Higgs boson production and decay rates and constraints on its couplings from a combined ATLAS and CMS analysis of the LHC 
pp collision data at $\sqrt{s}=7$ and $8 \mathrm{TeV}$, JHEP 08, 045 (2016). arXiv: 1606.02266

4. LHC Higgs Cross Section Working Group Collaboration, J. R. Andersen et al., Handbook of LHC Higgs Cross Sections: 3. Higgs Properties. arXiv: 1307.1347

5. LHC Higgs Cross Section Working Group Collaboration, D. de Florian et al., Handbook of LHC Higgs Cross Sections: 4. Deciphering the Nature of the Higgs Sector. arXiv:1610.07922

6. HiggsTools Working Group Collaboration, M. Boggia et al., The HiggsTools Handbook: Concepts and observables for deciphering the Nature of the Higgs Sector. arXiv:1711.09875

7. W. Buchmuller, D. Wyler, Effective Lagrangian analysis of new interactions and flavor conservation. Nucl. Phys. B 268, 621-653 (1986)

8. B. Grzadkowski, M. Iskrzynski, M. Misiak, J. Rosiek, Dimensionsix terms in the Standard Model Lagrangian. JHEP 10, 085 (2010). arXiv: 1008.4884

9. ATLAS Collaboration, G. Aad et al., Measurements of fiducial and differential cross sections for Higgs boson production in the diphoton decay channel at $\sqrt{s}=8 \mathrm{TeV}$ with ATLAS, JHEP 09, 112 (2014). arXiv:1407.4222

10. ATLAS Collaboration, G. Aad et al., Fiducial and differential cross sections of Higgs boson production measured in the four-lepton decay channel in $p p$ collisions at $\sqrt{s}=8 \mathrm{TeV}$ with the ATLAS detector, Phys. Lett. B 738, 234-253 (2014). arXiv:1408.3226

11. ATLAS Collaboration, G. Aad et al., Measurements of the Total and Differential Higgs Boson Production Cross Sections Combining the $H \rightarrow \gamma \gamma$ and $H \rightarrow Z Z^{*} \rightarrow 4 l$ Decay Channels at $\sqrt{s}=8$ ??TeV with the ATLAS Detector, Phys. Rev. Lett. 115(9), 091801 (2015). arXiv:1504.05833

12. ATLAS Collaboration, G. Aad et al., Measurement of fiducial differential cross sections of gluon-fusion production of Higgs bosons decaying to $\mathrm{WW}^{*} \rightarrow e v \mu \nu$ with the ATLAS detector at $\sqrt{s}=8$ TeV, JHEP 08, 104 (2016). arXiv:1604.02997

13. CMS Collaboration, S. Chatrchyan et al., Measurement of differential cross sections for Higgs boson production in the diphoton decay channel in pp collisions at $\sqrt{(} s)=8 \mathrm{TeV}$. arXiv:1508.07819 (2015). arXiv:1508.07819

14. CMS Collaboration, V. Khachatryan et al., Measurement of differential and integrated fiducial cross sections for Higgs boson production in the four-lepton decay channel in pp collisions at $\sqrt{s}=7$ and 8 TeV. JHEP 04, 005 (2016). arXiv: 1512.08377

15. CMS Collaboration, V. Khachatryan et al., Measurement of the transverse momentum spectrum of the Higgs boson produced in pp collisions at $\sqrt{s}=8 \mathrm{TeV}$ using $H \rightarrow W W$ decays. JHEP 03, 032 (2017). arXiv:1606.01522

16. ATLAS Collaboration, M. Aaboud et al., Measurement of inclusive and differential cross sections in the $H \rightarrow Z Z^{*} \rightarrow 4 \ell$ decay channel in $p p$ collisions at $\sqrt{s}=13 \mathrm{TeV}$ with the ATLAS detector, JHEP 10, 132 (2017). arXiv: 1708.02810

17. ATLAS Collaboration, M. Aaboud et al., Measurements of Higgs boson properties in the diphoton decay channel with $36 \mathrm{fb}^{-1}$ of $p p$ collision data at $\sqrt{s}=13 \mathrm{TeV}$ with the ATLAS detector. arXiv: 1802.04146

18. CMS Collaboration, A.M. Sirunyan et al., Measurements of properties of the Higgs boson decaying into the four-lepton final state in pp collisions at $\sqrt{s}=13$ TeV. JHEP 11, 047 (2017). arXiv:1706.09936

19. A. Banfi, A. Martin, V. Sanz, Probing top-partners in Higgs+jets. JHEP 08, 053 (2014). arXiv: 1308.4771

20. A. Azatov, C. Grojean, A. Paul, E. Salvioni, Resolving gluon fusion loops at current and future hadron colliders. JHEP 09, 123 (2016). arXiv: 1608.00977

21. R. Boughezal, F. Caola, K. Melnikov, F. Petriello, M. Schulze, Higgs boson production in association with a jet at next-to-next- to-leading order in perturbative QCD. JHEP 06, 072 (2013). arXiv: 1302.6216

22. R. Boughezal, F. Caola, K. Melnikov, F. Petriello, M. Schulze, Higgs boson production in association with a jet at next-tonext-to-leading order. Phys. Rev. Lett. 115(8), 082003 (2015). arXiv:1504.07922

23. X. Chen, T. Gehrmann, E.W.N. Glover, M. Jaquier, Precise QCD predictions for the production of Higgs + jet final states. Phys. Lett. B 740, 147-150 (2015). arXiv: 1408.5325

24. R. Bonciani, V. Del Duca, H. Frellesvig, J.M. Henn, F. Moriello, V.A. Smirnov, Two-loop planar master integrals for Higgs $\rightarrow 3$ partons with full heavy-quark mass dependence. JHEP 12, 096 (2016). arXiv: 1609.06685

25. K. Kudashkin, K. Melnikov, C. Wever, Two-loop amplitudes for processes $\mathrm{gg} \rightarrow \mathrm{Hg}, q g \rightarrow H q$ and $q \bar{q} \rightarrow H g$ at large Higgs transverse momentum. JHEP 02, 135 (2018). arXiv: 1712.06549

26. J .M. Lindert, K. Kudashkin, K. Melnikov, C. Wever, Higgs bosons with large transverse momentum at the LHC. Phys. Lett. B782, 210-214 (2018). arXiv:1801.08226

27. T. Neumann, NLO Higgs+jet at Large transverse momenta including top quark mass effects. arXiv: 1802.02981

28. S.P. Jones, M. Kerner, G. Luisoni, Next-to-leading-order QCD corrections to Higgs Boson Plus jet production with full Topquark mass dependence. Phys. Rev. Lett. 120(16), 162001 (2018). arXiv: 1802.00349

29. G. Brooijmans et al., Les Houches 2015: Physics at TeV collidersnew physics working group report. arXiv:1605.02684

30. R.V. Harlander, S. Liebler, H. Mantler, SusHi Bento: beyond NNLO and the heavy-top limit. Comput. Phys. Commun. 212, 239257 (2017). arXiv: 1605.03190

31. C. Anastasiou, C. Duhr, F. Dulat, E. Furlan, T. Gehrmann, F. Herzog, A. Lazopoulos, B. Mistlberger, CP-even scalar boson production via gluon fusion at the LHC. JHEP 09, 037 (2016). arXiv: 1605.05761

32. C. Grojean, E. Salvioni, M. Schlaffer, A. Weiler, Very boosted Higgs in gluon fusion. JHEP 05, 022 (2014). arXiv: 1312.3317

33. A. Azatov, A. Paul, Probing Higgs couplings with high $p \_T$ Higgs production. JHEP 01, 014 (2014). arXiv: 1309.5273

34. U. Langenegger, M. Spira, I. Strebel, Testing the Higgs Boson coupling to gluons (2015). arXiv:1507.01373. arXiv:1507.01373

35. R.V. Harlander, T. Neumann, Probing the nature of the Higgs-gluon coupling. Phys. Rev. D 88, 074015 (2013). arXiv:1308.2225

36. S. Dawson, I.M. Lewis, M. Zeng, Effective field theory for Higgs boson plus jet production. Phys. Rev. D 90(9), 093007 (2014). arXiv:1409.6299

37. D. Choudhury, P. Saha, Higgs production as a probe of anomalous top couplings. JHEP 08, 144 (2012). arXiv: 1201.4130

38. C. Degrande, J.M. Gerard, C. Grojean, F. Maltoni, G. Servant, Probing Top-Higgs non-standard interactions at the LHC. JHEP 07, 036 (2012). arXiv:1205.1065 [Erratum: JHEP03,032(2013)]

39. C. Zhang, Automating predictions for standard model effective field theory in MadGraph5 aMC@NLO, PoSRADCOR2015, 101 (2016). arXiv:1601.03994

40. F. Maltoni, E. Vryonidou, C. Zhang, Higgs production in association with a top-antitop pair in the Standard Model Effective Field Theory at NLO in QCD. JHEP 10, 123 (2016). arXiv:1607.05330

41. N. Deutschmann, C. Duhr, F. Maltoni, E. Vryonidou, Gluon-fusion Higgs production in the Standard Model Effective Field Theory. JHEP 12, 063 (2017). arXiv: 1708.00460

42. M. Grazzini, A. Ilnicka, M. Spira, M. Wiesemann, Modeling BSM effects on the Higgs transverse-momentum spectrum in an EFT approach. JHEP 03, 115 (2017). arXiv:1612.00283

43. G.F. Giudice, C. Grojean, A. Pomarol, R. Rattazzi, The strongly-interacting light Higgs. JHEP 06, 045 (2007). arXiv:hep-ph/0703164 
44. R. Contino, M. Ghezzi, C. Grojean, M. Muhlleitner, M. Spira, Effective Lagrangian for a light Higgs-like scalar. JHEP 07, 035 (2013). arXiv: 1303.3876

45. M. Grazzini, A. Ilnicka, M. Spira, M. Wiesemann, Effective Field Theory for Higgs properties parametrisation: the transverse momentum spectrum case, 52nd Rencontres de Moriond QCD 2017, La Thuile (2017). arXiv: 1705.05143

46. M. Grazzini, A. Ilnicka, M. Spira, M. Wiesemann, Effective field theory in quest to parametrise Higgs properties: the transverse momentum spectrum case. J. Phys. Conf. Ser. 873(1), 012050 (2017)

47. M. Spira, Radiative QCD corrections to decay and production of Higgs bosons at e+e- and pp accelerators (in German), PhD thesis, Aachen, Tech. Hochsch. (1993)

48. M. Spira, A. Djouadi, D. Graudenz, P.M. Zerwas, Higgs boson production at the LHC. Nucl. Phys. B 453, 17-82 (1995). arXiv:hep-ph/9504378

49. R.K. Ellis, I. Hinchliffe, M. Soldate, J.J. van der Bij, Higgs decay to tau+ tau-: a possible signature of intermediate Mass Higgs Bosons at the SSC. Nucl. Phys. B 297, 221 (1988)

50. J. Butterworth et al., PDF4LHC recommendations for LHC Run II. J. Phys. G 43,
023001 (2016). arXiv:1510.03865

51. NNPDF Collaboration, R. D. Ball et al., Parton distributions for the LHC Run II, JHEP 04, 040 (2015). arXiv:1410.8849

52. S. Dulat, T.-J. Hou, J. Gao, M. Guzzi, J. Huston, P. Nadolsky, J. Pumplin, C. Schmidt, D. Stump, C.P. Yuan, New parton distribution functions from a global analysis of quantum chromodynamics. Phys. Rev. D 93(3), 033006 (2016). arXiv:1506.07443

53. L.A. Harland-Lang, A.D. Martin, P. Motylinski, R.S. Thorne, Parton distributions in the LHC era: MMHT 2014 PDFs. Eur. Phys. J. C 75(5), 204 (2015). arXiv:1412.3989

54. J. Gao, P. Nadolsky, A meta-analysis of parton distribution functions. JHEP 07, 035 (2014). arXiv:1401.0013

55. S. Carrazza, S. Forte, Z. Kassabov, J.I. Latorre, J. Rojo, An unbiased Hessian representation for Monte Carlo PDFs. Eur. Phys. J. C 75(8), 369 (2015). arXiv: 1505.06736

56. D. Buarque Franzosi, C. Zhang, Probing the top-quark chromomagnetic dipole moment at next-to-leading order in QCD. Phys. Rev. D 91(11), 114010 (2015). arXiv:1503.08841

57. M. Spira, HIGLU: a program for the calculation of the total Higgs production cross-section at hadron colliders via gluon fusion including QCD corrections. arXiv:hep-ph/9510347 (1995) 